\title{
Recent experience of adult small-intestinal and multi-visceral transplantation at Addenbrooke's Hospital, Cambridge University, UK
}

\author{
A. Wiles ${ }^{1}$, A. Butler ${ }^{1}$, H. Tincknell ${ }^{1}$, S. Duncan ${ }^{1}$, B. Chukualim ${ }^{1}$, S. M. Gabe ${ }^{2}$, P. Gibbs ${ }^{1}$, \\ N. V. Jamieson ${ }^{1}$, R. K. Praseedom ${ }^{1}$, C. J. Watson ${ }^{1}$, J. M. Woodward ${ }^{1}$ and S. J. Middleton $^{1}$ \\ ${ }^{1}$ Addenbrooke's, Cambridge University Teaching Hospital, CB20QQ, UK and ${ }^{2}$ Lennard-Jones Intestinal Failure Unit, \\ St Mark's Hospital and Academic Institute, Harrow, UK
}

The number of adult patients undergoing small intestinal (SI) transplantation in the UK has increased. In Cambridge, 12 patients have received 13 small intestinal grafts either alone or as part of a composite graft, over the last 3 years. The primary diseases of the patients were Crohn's disease (x2), visceral myopathy (x3), desmoid disease (x2), mesenteric vascular disease (x2) severe adhesive disease resulting in subtotal enterectomy $(\mathrm{x} 1)$ and acute severe rejection $(\mathrm{x} 1)$. Indications for transplantation included loss of vascular access, parenteral nutrition-related liver disease, severe mesenteric arterial disease and acute severe rejection. Four patients received an isolated SI, 5 modified multi-visceral (multiple organs no liver) and 3 multi-visceral (1 patient received 2 grafts) (multiple organs including liver). Splenic and renal grafts were included in 1 and 4 patients, respectively.

Post-operative complications included infection, rejection, renal impairment and venous thrombosis. One patient who underwent splenic transplantation developed transient graft $v$. host disease and another had acute severe rejection requiring an urgent re-do multi-visceral graft which was successful. One patient was found to have an occult malignancy (gastric leiomyosarcoma) in the explant specimen and died a year later from metastatic disease, all other patients are alive. Overall survival to date is $92 \%$, and non-oncological survival is $100 \%$. All who have completed the early post-operative recovery are taking food orally as the sole source of nutrients with little if any dietary restriction (one patient recently transplanted remains on enteral feed supplements as per protocol). One patient has ongoing fatigue, 1 is undergoing treatment for CMV reactivation and another is recovering from pneumonia. All others have good or excellent overall function, 3 are considering conception, 1 regularly travels internationally.

Our recent experience is encouraging and similar to that of the best-performing centre in North America. The early survival of patients following intestinal transplantation is now excellent, graft function is usually complete and the majority of patients have good or excellent overall function. We consider that it is imperative to refer patients for assessment before they have developed a degree of co-morbidity that precludes transplantation. All patients referred will be discussed in detail at the National adult intestinal transplantation forum (NASIT) by a multidisciplinary team from several intestinal failure and transplantation centres before being considered for transplantation. Clinicians are welcome to attend the Forum. 